\title{
Correspondence
}

\section{Risk assessment: dealing with uncertainty}

Large et al's article on the predictive value of risk categorisation in schizophrenia ${ }^{1}$ is an elegantly written and sobering analysis on clinical risk assessment practices. Their arguments that risk categorisation approaches are limited and may sometimes do more harm than good are reminders of the limitations of the risk management approach. The imperfect nature of risk categorisation is compellingly demonstrated by application of their hypothetical instrument for risk calculation (HIRC) model, applied to the best data available and in a manner that is, if anything, giving risk categorisation the fairest of road tests. Clinical risk assessments, be they based on clinical expertise, structured clinical assessment or actuarial tools, are limited because of the mathematics of low frequency events.

In 'Probability and loss: two sides of the risk assessment coin', ${ }^{2}$ Large \& Nielssen advance their concerns about the current practice of risk management by examining the loss element of the risk assessment equation and the current limitation of any instrument to allow for multiplication of the sundry risks that may occur in the course of an unfolding episode of mental illness. They also point out, quite correctly, that clinicians are often operating on limited information. Our own experience with poor handover of all the available clinically important information (from referring clinicians, and family, or medical records stored in another facility) reminds us that even if we had the perfect tool, the risk assessment will only be as good as the information used will allow.

These papers will be disconcerting for many clinicians and managers. The changeability of risk and elements of uncertainty in the human interaction of the assessment are other limitations. ${ }^{3}$ Added to this is the nature of the task of assessing a person whose illness, personality or state of mind may be constraints to accurate assessment. People may conceal information or their true feelings for a variety of reasons.

These arguments against a risk assessment approach to managing clinical risk are important in ensuring against complacency and provide impetus for continuing development and refinement of our clinical practice. However, we need also to acknowledge that this is a discussion which is inevitably grounded in a number of frameworks other than the statistical. In their articles, Large and Nielssen have not made reference to the moral, legal, ethical, cultural, political, compassionate and most importantly pragmatic frames of reference that support the argument for continuing to practise a risk assessment approach. ${ }^{1,2}$ Although their approach is welcome in the arena of scientific discussion, it does not wash in the real world. Winston Churchill famously espoused the view that democracy 'is the worst form of government except all those others that have been tried'. So it is with risk assessment in our current time.

The risk management approach when undertaken properly includes participation from a number of stakeholders, including the patient, family, and health professionals, in efforts to reduce or mitigate risk factors that are drawn from larger population studies, from information available in the clinical encounter and from collateral sources. Assessing risk is a task inherent in psychiatric assessment, and its importance lies less in the assignation of a category of risk (high, low) than in the way the risks identified inform a treatment or management plan. The plan will ideally include the set of indicated interventions, delivered within an expected timeframe, that are considered best to manage and reduce the risks. There will always be uncertainty whether any risk will eventuate, even for those thought to have a high level of risk. As Large et al point out $^{1}{ }^{1}$ this will leave a larger number of people who are judged as low risk with no intervention (above standard care), some of whom will turn out to have an adverse event.

We are well served if this discussion reminds clinicians, patients and families that we have no perfect powers of prediction and draws the attention of researchers and clinical experts to reach for the next innovation to our methods.

To close, let us quote from P. Bernstein: 'The essence of risk management lies in maximising the areas where we have some control over the outcome while minimising the areas where we have absolutely no control over the outcome and the linkage between effect and cause is hidden from us. ${ }^{4}$

1 Large MM, Ryan CJ, Singh SP, Paton MB, Nielssen OB. The predictive value of risk categorisation in schizophrenia. Harv Rev Psychiatry 2011; 19: $25-33$

2 Large MM, Nielssen OB. Probability and loss: two sides of the risk assessment coin. Psychiatrist 2011; 35: 413-8.

3 O'Connor N, Warby M, Raphael B, Vassallo T. Changeability, confidence, common sense and corroboration: comprehensive suicide risk assessment. Australas Psychiatry 2004; 12: 352-60.

4 Bernstein P. Against the Gods: The Remarkable Story of Risk. John Wiley \& Sons, 1996.

Nick O'Connor is Clinical Senior Lecturer at the Department of Psychological Medicine, University of Sydney, Australia, email: nick.oconnor@sswahs.nsw.gov.au, and Scott Clark is Associate Professor at the School of Medicine and Public Health, University of Newcastle, Australia.

doi: 10.1192/pb.36.2.78

\section{What level of risk is acceptable in psychiatry?}

The review of risk assessment by Large $\&$ Nielssen ${ }^{1}$ is timely as there has been an increasing tendency to rely on structured protocols in the assessment of patients, particularly with regard to future probabilities of violence and self-harm. However, there are a number of aspects which have not been discussed, the most important of these being the concept of acceptable risk.

Politicians and service managers are happy to point to a process of risk assessment, yet they universally abrogate their duty as representatives of the community to define what level of risk is acceptable. Despite the statistical difficulties discussed by Large \& Nielssen, this has been successfully embraced in aviation medicine. Acceptable failure rates in mechanical components have been used to define the risk management for pilot incapacitation. Although this approach was pioneered by cardiologists, aviation medicine is where the approach has been more widely adopted, even though the 